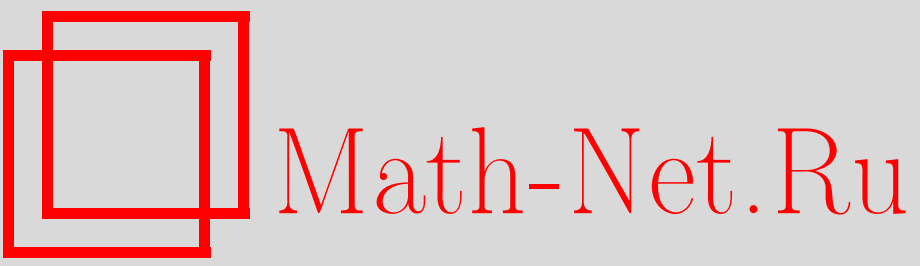

М. И. Вязовский, Н. А. Кивель, Анализ аксиальной аномалии с помощью проекционной техники в размерной регуляризации, ТМФ, 1996, том 109, номер 3, 372380

DOI: https://doi.org/10.4213/tmf1234

Использование Общероссийского математического портала Math-Net.Ru подразумевает, что вы прочитали и согласны с пользовательским соглашением http://www.mathnet.ru/rus/agreement

Параметры загрузки:

IP: 54.84 .234 .179

26 апреля 2023 г., 12:36:35 
ТЕОРЕТИЧЕСКАЯ

И МАТЕМАТИЧЕСКАЯ

ФИЗИКА

Том 109, № 3

декабрь, 1996

М.И. Вязовский* , Н. А. Кивель*

\section{АНАЛИЗ АКСИАЛЬНОЙ АНОМАЛИИ С ПОМОШЬЮ ПРОЕКЦИОННОЙ ТЕХНИКИ В РАЗМЕРНОЙ РЕГУЛЯРИЗАЦИИ}

Предлагается способ представления киральной аномалии в рамках размерной регуляризации без использования символов $\gamma_{5}$ и $\varepsilon_{\mu \nu \lambda \rho}$ в нецелой размерности. Этот способ применяется для расчета и анализа ренормировки киральной аномалии в безмассовой квантовой электродинамике.

\section{1. ВВЕДЕНИЕ}

Обшепризнано, что размерная регуляризация и минимальные выгитания - MS - одна из самых удобных вычислительных схем. Однако известно, что в задачах со спинорными полями есть проблемы с размерной регуляризацией, если приходится иметь дело с символами $\varepsilon_{\mu \nu \lambda \rho}$ и $\gamma_{5}=\varepsilon_{\mu \nu \lambda \rho} \gamma_{\mu} \gamma_{\nu} \gamma_{\lambda} \gamma_{\rho} / 4$ !. Простейший пример - расчет коэффициента $a$ аксиальной аномалии

$$
\partial_{\nu} J_{5}^{\nu}=a \varepsilon_{\mu \nu \lambda \rho} F_{\mu \nu} F_{\lambda \rho}
$$

в безмассовой квантовой электродинамике (объекты в (1) понимаются как ренормированные составные операторы). В литературе предложено много разных способов расчета аксиальной аномалии в размерной регуляризации $[1,2,3]$. В данной работе мы хотим предложить еше один способ расчета, на наш взгляд, достаточно простой и естественньй. Основная идея состоит в том, что киральные преобразования в размерности $D=4$ можно естественно продолжить в произвольную размерность $D$ без использования символов $\varepsilon_{\mu \nu \lambda \rho}$ и $\gamma_{5}$, и это позволяет выполнить все выкладки по обычным правилам. Такой расчет в схеме MS с двухпетлевой точностью дает

$$
a=N u+4 N u^{2}+O\left(u^{3}\right)
$$

где $u=e^{2} / 16 \pi^{2}$ - заряд, $N$ - число изотопических компонент спинорного поля. В ответе (2), в отличие от формулы, приведенной в [4], есть двухпетлевой вклад (видимо, и все следующие), что связано с различием схем вычитаний. Ответ (2) получен путем анализа ренормированного тождества Уорда (отметим, что мы при этом работаем только с самими составными операторами, а не с их матричными элементами) и затем проверен с помощью уравнений ренормгруппы в духе работы [2]. Важную роль при получении равенства (1) для $D=4$ играют формулы проекционной техники $[3,5,6]$ для составных операторов.

* Петербургский институт ядерной физики им. Б. П. Константинова 


\section{2. КИРАЛЬНОЕ ПРЕОБРАЗОВАНИЕ В РАЗМЕРНОСТИ $D=4-2 \varepsilon$, ТОЖДЕСТВА УОР ДА}

Мы будем рассматривать евклидову безмассовую $U_{N}$-симметричную квантовую электродинамику в размерности $D=4-2 \varepsilon$, записывая неренормированную плотность лагранжиана $\mathcal{L}_{0}=\mathcal{L}_{0}\left(\phi, e_{0}, \alpha_{0}\right)$ в виде

$$
\mathcal{L}_{0}=-\frac{1}{4} F_{\mu \nu} F_{\mu \nu}-\frac{1}{2 \alpha_{0}}\left(\partial_{\mu} A_{\nu}\right)^{2}+\bar{\psi}^{a}\left(\stackrel{\widehat{\leftrightarrow}}{\partial}+i e_{0} \widehat{A}\right) \psi^{a},
$$

где $\phi=\left(\bar{\psi}, \psi, A_{\mu}\right)$ - набор всех полей, $e_{0}$ и $\alpha_{0}$ - затравочные параметры, $F_{\mu \nu}=\partial_{\mu} A_{\nu}-$ $\partial_{\nu} A_{\mu}, \stackrel{\leftrightarrow}{\partial}=\frac{1}{2}(\partial-\overleftarrow{\partial})$. Изотопический индекс $a=1, \ldots, N$ у спинорных полей в дальнейшем будем опускать, подразумевая суммирование по нему во всех квадратичных формах $\bar{\psi} \cdots \psi$. Из-за отсутствия в (3) символов $\varepsilon_{\mu \nu \lambda \rho}$ и $\gamma_{5}$ переменность $D$ не порождает проблем с $\gamma$-матрицами, модель (3) в размерности $D=4-2 \varepsilon$ является мультипликативно ренормируемой, ее ренормированное действие имеет стандартный вид

$$
S_{R}(\phi)=\int d^{D} x \mathcal{L}_{0}\left(\phi Z_{\phi}, e_{0}=e \mu^{\varepsilon} Z_{e}, \alpha_{0}=\alpha Z_{\alpha}\right)
$$

где $\mu$ - ренормировочная масса, $e$ и $\alpha$ - ренормированные параметры, $Z_{\phi, e, \alpha}$ - константы ренормировки, причем $Z_{A}=Z_{e}^{-1}$ и $Z_{\alpha}=Z_{A}^{2}$ в силу калибровочной инвариантности $[3,7]$.

При $D=4$ имеют смысл символы $\varepsilon_{\mu \nu \lambda \rho}$ и $\gamma_{5}=\varepsilon_{\mu \nu \lambda \rho} \gamma_{\mu} \gamma_{\nu} \gamma_{\lambda} \gamma_{\rho} / 4$ ! и модель (3) инвариантна относительно глобальных киральных преобразований $\psi \rightarrow\left(\exp \omega \gamma_{5}\right) \psi$, $\bar{\psi} \rightarrow \bar{\psi}\left(\exp \omega \gamma_{5}\right)$ спинорных полей. В инфинитезимальной форме им соответствует

$$
\delta_{\omega} \psi=\omega \gamma_{5} \psi, \quad \delta_{\omega} \bar{\psi}=\bar{\psi} \omega \gamma_{5},
$$

где $\omega$ - инфинитезимальньй параметр.

Мы хотим прежде всего обобшить преобразование (5) на случай переменной размерности $D=4-2 \varepsilon$. Напомним кратко известную технику работы с $\gamma$-матрицами (правильнее было бы говорить " $\gamma$-символами") в переменной размерности $D$. Тогда можно пользоваться только основным антикоммутационным соотношением

$$
\gamma_{\mu} \gamma_{\nu}+\gamma_{\nu} \gamma_{\mu}=2 \delta_{\mu \nu}
$$

которое позволяет привести любое произведение $\gamma$-матриц к линейной комбинации "базисных структур" - полностью антисимметризованных произведений

$$
\gamma_{A}^{(n)}=\gamma_{\left[\mu_{1}\right.} \ldots \gamma_{\left.\mu_{n}\right]} .
$$

Здесь $[\cdots]$ обозначает полную антисимметризацию по перестановкам всех индексов внутри скобок [ ], $A=\left(\mu_{1} \ldots \mu_{n}\right)$-компактное обозначение набора всех этих индексов.

Подчеркнем, что объекты (7), в отличие от $\gamma_{5}$, можно считать хорошо определенными в переменной размерности $D$, и правила работы с ними известны $[5,8]$. В целой размерности $D=4$ отличны от нуля лишь структуры (7) с $n \leq 4$, все следуюшие исчезают. Имея в виду в дальнейшем случай $D=4-2 \varepsilon$, будем называть структуры (7) с $n \leq 4$ 
младшими, а с $n>4$ - старшими. Те же термины будем употреблять и для составных операторов типа $\bar{\psi} \cdots \psi$, содержаших внутри выражения (7): старшими считаются те операторы, которые формально исчезают при $D=4$ в силу исчезновения входящих в них старших структур (7).

Обобщением (5) на случай $D=4-2 \varepsilon$ естественно считать преобразования

$$
\delta_{\omega} \psi=\omega_{A} \gamma_{A}^{(4)} \psi, \quad \delta_{\omega} \bar{\psi}=\omega_{A} \bar{\psi} \gamma_{A}^{(4)}
$$

с инфинитезимальным параметром $\omega_{A}$, полностью антисимметричным по набору четыpeх индексов $A=\left(\mu_{1} \mu_{2} \mu_{3} \mu_{4}\right)$. Ясно, что при $D=4$ преобразования (8) переходят в (5), но они сохраняют смысл и в нецелой размерности, хотя соответствуюшая симметрия в (3) будет явно нарушенной.

Независимо от наличия симметрии по данной плотности лагранжиана $\mathcal{L}$ можно построить соответствующий преобразованиям (8) нетеровский ток. Для плотности (3) получим

$$
J_{A}^{\nu}=\frac{1}{2} \bar{\psi}\left[\gamma_{\nu}, \gamma_{A}^{(4)}\right] \psi \equiv 4 \bar{\psi}\left(\delta_{\nu\left[\mu_{1}\right.} \gamma_{\left.\mu_{2} \mu_{3} \mu_{4}\right]}^{(3)}\right) \psi
$$

а для ренормированной модели (4) появится дополнительный множитель $Z_{\psi}^{2}$. Отметим, что явный вид тока зависит от произвола типа полной производной в $\mathcal{L}$, не влияющего на функционал действия. Ток (9) калибровочно-инвариантен и $C$-четен, для обеспечения последнего свойства и была выполнена антисимметризация $\widehat{\partial} \rightarrow \overleftrightarrow{\partial}$ в $(3)$.

Для модели (3) (и неренормированной, и ренормированной) можно написать соответствуюшее (8) тождество Уорда

$$
\partial_{\nu} J_{A}^{\nu}+\delta_{A} S+N_{A}=0
$$

в котором $\delta_{A} S$ - вариация действия, в нашем случае имеем

$$
\delta_{A} S=\bar{\psi} \gamma_{A}^{(4)} \frac{\vec{\delta} S}{\delta \bar{\psi}}+\frac{\overleftarrow{\delta} S}{\delta \psi} \gamma_{A}^{(4)} \psi
$$

а $N_{A}$ - "нарушаюший" (данную симметрию) оператор. Для неренормированной плотности лагранжиана (3) получаем

$$
N_{A}=-2 \bar{\psi} \gamma_{A \nu}^{(5)}\left(\overleftrightarrow{\partial}_{\nu}+i e_{0} A_{\nu}\right) \psi=\partial_{\nu}\left(\bar{\psi} \gamma_{A \nu}^{(5)} \psi\right)-2 \bar{\psi} \gamma_{A \nu}^{(5)}\left(\partial_{\nu}+i e_{0} A_{\nu}\right) \psi
$$

а для ренормированной модели (4) в (12) произойдет замена $e_{0} \rightarrow e \mu^{\varepsilon}$ и добавится общий множитель $Z_{\psi}^{2}$. Отметим, что $(12)$ - старший оператор, поэтому при $D=4$ он исчезает и симметрия восстанавливается. Однако из-за УФ-расходимостей переход от $D=4-2 \varepsilon$ к $D=4$ корректен только для ренормированных составных операторов, допускающих предел $\varepsilon \rightarrow 0$.

\section{3. РЕНОРМИРОВКА СОСТАВНЫХ ОПЕРАТОРОВ В ТОЖДЕСТВЕ УОР ДА И АКСИАЛЬНОЙ АНОМАЛИИ}

Приведем, следуя [3,9], только самые необходимые для дальнейшего сведения о ренормировке составных операторов, дополнительная информация дается в приложении. 
Говоря о составных операторах, нужно различать классические мономы или полиномы $F=F(\phi)$, построенные из классических полей $\phi$ (в нашем случае $\left.\phi=\left(\bar{\psi}, \psi, A_{\mu}\right)\right)$, и аналогичные операторные конструкции $\widehat{F}=F(\hat{\phi})$, в которых классический аргумент $\phi$ заменяется гайзенберговским оператором $\hat{\phi}$ (неренормированным) или $\hat{\phi}_{R}=Z_{\phi}^{-1} \hat{\phi}$ (ренормированным). При ренормировке могут смешиваться между собой лишь операторы с одинаковой размерностью и симметрией, полный набор таких операторов образует “замкнутую систему". Если $F_{i}(\phi)(i=1,2, \ldots)$ - такая система неренормированных операторов, а $\left[F_{i}(\phi)\right]_{R}$ - соответствуюшие ренормированные классические операторы (см. приложение), то соотношения

$$
\left[F_{i}(\phi)\right]_{R}=\sum_{k} Q_{i k} F_{k}(\phi), \quad i=1,2, \ldots
$$

определяют матрицу смешивания $Q$. B схеме MS диагональные элементы $Q_{i i}$ имеют вид $1+$ полюсы по $\varepsilon$, а недиагональные содержат только полюсы, поэтому У $\Phi$-конечной частью $F_{i}(\phi)$ является просто $\left[F_{i}(\phi)\right]_{R}$. Неренормированные $\left(\widehat{F}_{i}\right)$ и ренормированные $\left(\widehat{F}_{i R}\right)$ составные операторы определяются по классическим функционалам соотношениями

$$
\widehat{F}_{i}=\left.F_{i}(\phi)\right|_{\phi=\hat{\phi}}, \quad \widehat{F}_{i R}=\left.\left[F_{i}(\phi)\right]_{R}\right|_{\phi=\hat{\phi}_{R}}
$$

и связаны между собой матрицей ренормировки $Z_{F}$ :

$$
\widehat{F}_{i}=\sum_{k}\left(Z_{F}\right)_{i k} \widehat{F}_{k R}
$$

Матрица $Z_{F}$ выражается через матрицу $Q$ в (13) и константы ренормировки полей $Z_{\phi}$, по $Z_{F}$ известным образом $[3,9]$ находится матрица аномальных размерностей данного семейства составных операторов.

В тождество Уорда (10) входят $C$-четные калибровочно-инвариантные операторы с канонической размерностью 4 (при $\varepsilon=0$ ). В качестве полного базиса этого семейства удобно взять следующие три оператора:

$$
F_{1}=\partial_{\nu} J_{A}^{\nu}, \quad F_{2}=F_{\left[\mu_{1} \mu_{2}\right.} F_{\left.\mu_{3} \mu_{4}\right]}, \quad F_{3}=N_{A}
$$

с обычным тензором $F_{\mu \nu}$, током (9) и нарушаюшим оператором (12). Все операторы (16) зависят от четырех внешних индексов $A=\left(\mu_{1} \mu_{2} \mu_{3} \mu_{4}\right)$ и полностью антисимметричны по ним, что для $F_{2}$ обозначается через [...].

Три оператора (16) смешиваются при ренормировке только между собой. Соответствуюшая 3 × 3-матрица смешивания (13) имеет следуюшую структуру:

$$
Q=\left(\begin{array}{ccc}
\times & 0 & 0 \\
\times & \times & 0 \\
\times & \times & \times
\end{array}\right),
$$

где крестиками обозначены ненулевые (в общем случае) элементы. Такой вид $Q$ объясняется тем, что $F_{1,2}$ в $(16)$ являются полными производными (для $F_{2}$ это видно из тождества $\left.F_{\left[\mu_{1} \mu_{2}\right.} F_{\left.\mu_{3} \mu_{4}\right]}=4 \partial_{\nu}\left\{\delta_{\nu\left[\mu_{1}\right.} A_{\mu_{2}} \partial_{\mu_{3}} A_{\left.\mu_{4}\right]}\right\}\right)$, а $F_{3}$ - нет, поэтому $F_{1,2}$ могут смешиваться только между собой. Соответствуюший $2 \times 2$-блок (17) определяется смешиванием пятизначковых операторов под знаком $\partial_{\nu}$, имеюших размерность 3 . Первый из них 
$\left(J_{A}^{\nu}\right)$ калибровочно-инвариантен, второй - нет, чем и объясняется равенство $Q_{12}=0$ B (17).

Информацию о третьей строке матрицы (17) можно получить из тождества Уорда (10) для ренормированного действия (4). В обозначениях (16) оно принимает вид

$$
Z_{\psi}^{2}\left(F_{1}+F_{3}\right)+\delta_{A} S_{R}=0
$$

Поскольку $\delta_{A} S_{R}-$ У $\Phi$-конечный оператор [3, 9], должна быть УФ-конечной и сумма прочих вкладов в (18), следовательно, она должна совпадать со своей У $\Phi$-конечной частью. Для ренормировки в схеме MS отбор УФ-конечной части сводится к замене $Z \rightarrow 1$ и $F \rightarrow[F]_{R}$, откуда получаем

$$
Z_{\psi}^{2}\left(F_{1}+F_{3}\right)=\left[F_{1}\right]_{R}+\left[F_{3}\right]_{R}
$$

Если выразить здесь $[F]_{R}$ через $F$ с помощью (13) и затем приравнять коэффициенты при всех $F_{i}$ в обеих частях равенства, получим следующие выражения для элементов третьей строки матрицы (17) в схеме MS:

$$
Q_{31}=Z_{\psi}^{2}-Q_{11}, \quad Q_{32}=0, \quad Q_{33}=Z_{\psi}^{2}
$$

Из (18) и (19) получаем ренормированную форму тождества Уорда

$$
\left[F_{1}\right]_{R}+\left[F_{3}\right]_{R}+\delta_{A} S_{R}=0 .
$$

При замене $\phi \rightarrow \hat{\phi}_{R}$ вклад $\delta_{A} S_{R}$ в $(21)$ исчезает (поскольку $\hat{\phi}_{R}$ удовлетворяет классическим уравнениям движения для действия $\left.S_{R}(\phi)\right)$, так что равенство $(21)$ в терминах ренормированных операторов принимает вид

$$
\widehat{F}_{1 R}+\widehat{F}_{3 R}=0
$$

Ренормированные операторы $\widehat{F}_{R}$ и их классические аналоги $[F]_{R}$, в отличие от неренормированных, имеют смысл и при $\varepsilon=0$, и тождество Уорда (22) при $\varepsilon=0$ должно привести к выражению (1) для аксиальной аномалии. Чтобы убедиться в этом, нужно воспользоваться соотношениями проекционной техники $[3,5]$. Обшее правило состоит в том, что для любой замкнутой системы смешиваюшихся (и независимых при $\varepsilon \neq 0$ ) составных операторов полный базис при $\varepsilon=0$ образуют только младшие, т.е. любой старший есть тогда некоторая линейная комбинация младших. Доказательством является сушествование [6] некоторой отличной от MS схемы ренормировки $\mathrm{MS}^{\prime}$ (см. приложение), совпадаюшей с обычной схемой MS для всех младших операторов и обеспечивающей исчезновение всех старших операторов при $\varepsilon=0$ :

$$
\left.\widehat{F}_{R}^{\prime}\right|_{\text {мл }}=\left.\widehat{F}_{R}\right|_{\text {мл }} \text { при } \forall \varepsilon,\left.\quad \widehat{F}_{R}^{\prime}\right|_{\text {ст }}=0 \text { при } \varepsilon=0 .
$$

С другой стороны, как известно, полученные в любых двух схемах (у нас MS и $\mathrm{MS}^{\prime}$ ) ренормированные операторы должны быть связаны между собой УФ-конечной ренормировкой, т.е.

$$
\widehat{F}_{i R}=\sum_{k} \bar{Z}_{i k} \widehat{F}_{k R}^{\prime}
$$


с некоторой УФ-конечной матрицей $\bar{Z}$.

Сказанное выше имеет прямое отношение к нашей задаче, т.к. в системе (16) операторы $F_{1,2}$ младшие, а $F_{3}$ старший. Поэтому для ренормированных в схеме MS операторов (16) при $\varepsilon=0$ должно выполняться равенство

$$
\widehat{F}_{3 R}=c_{1} \widehat{F}_{1 R}+c_{2} \widehat{F}_{2 R},
$$

в котором $c_{k}=\bar{Z}_{3 k}(k=1,2)$ - не зависяшие от $\varepsilon$ "проекционные коэффициенты".

Подставив $(25)$ в $(22)$, при $\varepsilon=0$ получим $\left(1+c_{1}\right) \widehat{F}_{1 R}+c_{2} \widehat{F}_{2 R}=0$, откуда следует

$$
\widehat{F}_{1 R}=-\frac{c_{2}}{1+c_{1}} \widehat{F}_{2 R} .
$$

Свернув это равенство по индексам $A=\left(\mu_{1} \mu_{2} \mu_{3} \mu_{4}\right)$ операторов (16) с тензором $\varepsilon_{A}$, при учете равенства $\varepsilon_{A} J_{A}^{\nu}=4 ! J_{5}^{\nu}$ (только для $D=4$ ) получим соотношение (1) для ренормированных операторов с

$$
a=-\frac{c_{2}}{24\left(1+c_{1}\right)} .
$$

Тем самым коэффициент $a$ в выражении (1) для аксиальной аномалии выражается через проекционные коэффициенты $c_{1,2}$ в $(25)$.

\section{4. РАСЧЕТ ПРОЕКЦИОННЫХ КОЭФФИЦИЕНТОВ}

Пусть $Q$ - матрица смешивания (17) для операторов (16) в схеме MS, $Q^{\prime}$ - аналогичная матрица для схемы $\mathrm{MS}^{\prime}$. Обе вычисляются по теории возмушений в форме рядов по заряду $u=e^{2} / 16 \pi^{2}$, причем $Q$ имеет вид $1+$ полюсы по $\varepsilon$, а в $Q^{\prime}$, кроме полюсов, есть вклады нулевого порядка по $\varepsilon$ в элементах $Q_{31}^{\prime}$ и $Q_{32}^{\prime}$ (см. приложение). Обозначив эти константы через $c_{3 k}(k=1,2)$, имеем $Q^{\prime}=1+c+$ полюсы по $\varepsilon$, где $c$ - не зависяшая от $\varepsilon$ матрица с указанными элементами $c_{3 k}$, прочие - нули.

Из определения (14) следует, что связь (24) сохраняет тот же вид и для классических операторов $[F]_{R}$ и $[F]_{R}^{\prime}$. С учетом (13) это приводит к матричному равенству $Q=\bar{Z} Q^{\prime}$ и его следствию

$$
\bar{Z}^{-1}=Q^{\prime} Q^{-1} .
$$

Учитывая обший вид матриц $Q$ и $Q^{\prime}$ (см. вьше), нетрудно убедиться, что в рамках теории возмущений правая часть $(28)$ имеет вид $1+c+$ полюсы по $\varepsilon$. Поскольку все это выражение должно быть У $\Phi$-конечным, все полюсы должны взаимно сокрашаться, поэтому

$$
\bar{Z}^{-1}=1+c .
$$

Тем самым доказано, что У $\Phi$-конечная матрица в (24) от $\varepsilon$ вообще не зависит (отметим аналогию с доказательством независимости от $\varepsilon$ аномальных размерностей в схеме MS $[3,9])$. Учитывая вид матрицы $c$ (см. выше), из (29) находим $\bar{Z}=1-c$, откуда получаем удобное для практических вычислений представление

$$
c_{k}=-c_{3 k} \equiv-\text { "УФ-конечная часть } Q_{3 k}^{\prime} ", \quad k=1,2,
$$




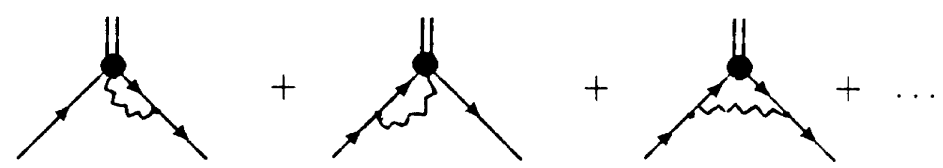

Рис. 1. Сумма диаграмм, дающих вклад в $c_{1}$.

для проекционных коэффиициентов $c_{k}=\bar{Z}_{3 k}$ в (25).

Коэффициенты (30) вычисляются по диаграммам 1-неприводимых функций Грина со вставкой оператора $F_{3}$ в схеме $\mathrm{MS}^{\prime}$ (см. приложение). Для получения величины (27) с двухпетлевой точностью коэффициент $c_{2}$ следует вычислить в двухпетлевом, а $c_{1}-$ в однопетлевом приближении. Все нужные для такого расчета диаграммы показаны на рис. 1 и 2, жирной точкой в этих графиках показана вершина составного оператора $F_{3}$. Расчет дает

$$
c_{1}=-4 u+O\left(u^{2}\right), \quad c_{2}=-24 N u+O\left(u^{3}\right),
$$

что вместе с (27) приводит к ответу (2) для $a$. Отметим, что все вклады двухпетлевых диаграмм в $c_{2}$ взаимно сокрашаются, так что двухпетлевой вклад в (2) порождается только поправкой от $c_{1}$ в знаменателе (27).
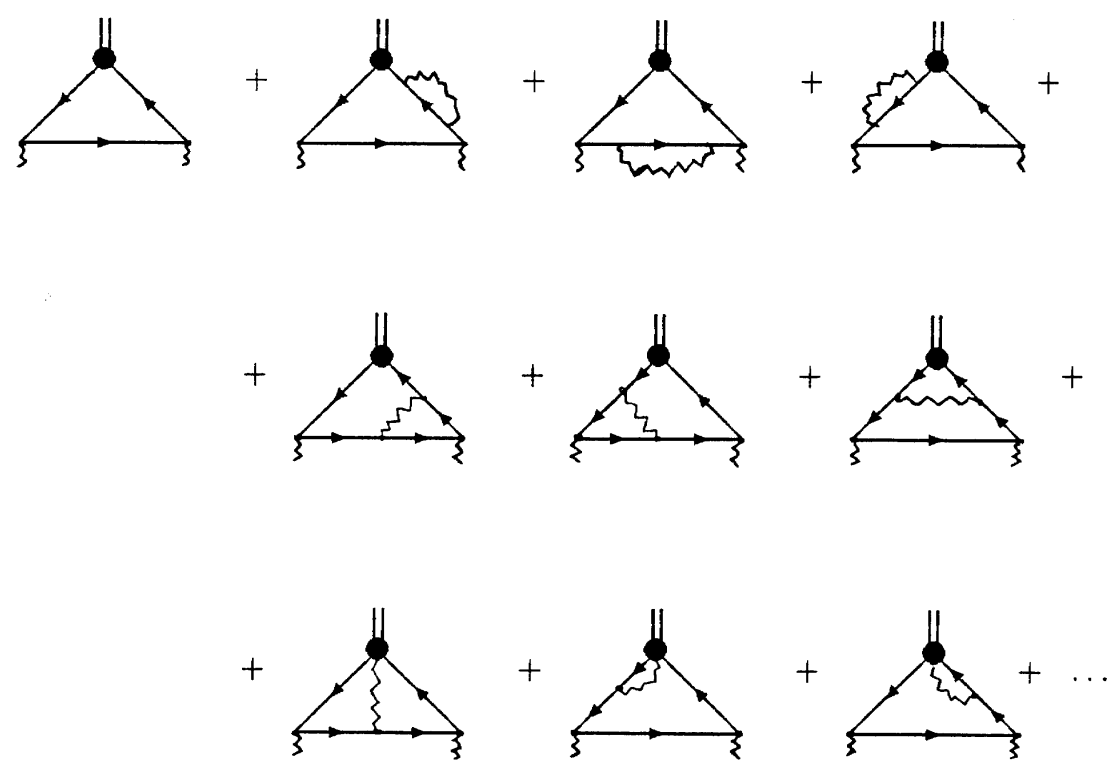

Рис. 2. Сумма диаграмм, дающих вклад в $c_{2}$.

Ответ (2) можно проверить с помошью уравнений ренормгруппы [2]. Рассмотрим равенство $(26)$ при $\varepsilon=0$, переписав его в виде $\widehat{F}_{1 R}=24 a \widehat{F}_{2 R}$, подействуем на обе части 
операцией $\mathcal{D}_{\text {РГ }}$ (равной $\mu \frac{d}{d \mu}$ при фиксированных затравочных параметрах) и затем исключим все операторы $\widehat{F}_{R}$ с помошњю исходного соотношения $\widehat{F}_{1 R}=24 a \widehat{F}_{2 R}$. Это дает

$$
\beta(u) \frac{d}{d u} a+\left(\gamma_{11}-\gamma_{22}\right) a-24 \gamma_{21} a^{2}=0 .
$$

При получении равенства (32) использованы соотношения $\mathcal{D}_{\mathrm{P} Г} a=\beta \frac{d}{d u} a$ и $\mathcal{D}_{\mathrm{P \Gamma}} \widehat{F}_{i R}=$ $-\sum_{k} \gamma_{i k} \widehat{F}_{k R}$, в которых $\beta(u)$ - бета-функция заряда $u$, а $\gamma_{i k}$ - элементы $2 \times 2$-матришы аномальных размерностей операторов $F_{1,2}$ в схеме MS (напомним, что $F_{1,2}$ смешиваются только между собой, причем $\gamma_{12}=0$ из-за треугольности соответствуюшего блока в (17)). Матрица $\gamma$ вычисляется по соответствующей матрице $Q$ и константам ренормировки полей в (4) [9], расчет с нужной точностью дает $\gamma_{11}=40 N u^{2} / 3+\cdots$, $\gamma_{22}=\beta_{1} u+\beta_{2} u^{2}+\cdots, \gamma_{21}=u+\cdots$, где $\beta_{1,2}$ - известные [10] первые коэффициенты в разложении бета-функции $\beta(u)=\beta_{1} u^{2}+\beta_{2} u^{3}+\cdots, \beta_{1}=8 N / 3, \beta_{2}=8 N$ (отметим, что $\beta$ и $\gamma_{i k}$ калибровочно-инвариантны).

Первый коэффициент $a_{1}$ в разложении $a=a_{1} u+a_{2} u^{2}+\cdots$ из (32) не определяется, но все следуюшие однозначно находятся по $a_{1}$ и ренормализационным функциям $\beta$ и $\gamma_{i k}$. Легко проверить, что двухпетлевой вклад (2) согласуется с (32) при указанных выше значениях $\gamma_{i k}$ (мы перепроверили их вычисление). Таким образом, ответ (2) для коэффициента аксиальной аномалии в схеме MS согласуется с уравнениями ренормгруппы.

Выражаем благодарность А. Н. Васильеву и А. С. Степаненко за многочисленные обсуждения и помощь в написании текста статьи.

Работа выполнена при поддержке Российского фонда фундаментальных исследований (грант № 96-02-17033) и Международного фонда фундаментальной физики в Москве в рамках проекта INTAS.

ПРИЛОЖЕНИЕ

\section{ОПЕРАТОРНЫЕ КОНТРЧЛЕНЫ В СХЕМАХ МS и МS'}

Матрища смешивания $Q$ в (13) выгисляется по диаграммам 1-неприводимых функций Грина со вставками операторов $F_{i}$ в базовой теории (т.е. ренормированной с заменой $Z \rightarrow 1$ для всех констант ренормировки). Ренормированный оператор $[F(\phi)]_{R}$, соответствующий данному неренормированному $F(\phi)$ (у всех операторов подразумевается свободньй аргумент $x$ ), определяется соотношением [9]

$$
[F(\phi)]_{R}=F(\phi)-L \Gamma_{F}(\phi),
$$

в котором $L$ - контрчленная операция выбранной ренормировочной схемы, $\Gamma_{F}(\phi)$-производяший функционал 1-неприводимых функций Грина с одним оператором $F(\phi)$ и любым числом полей $\phi$ в базовой теории. Операторные контрчлены $L \Gamma_{F}(\phi)$ в (П.1) являются линейными комбинациями самого $F$ и всех прочих независимых операторов с той же размерностью (при $\varepsilon=0$ ) и симметрией. Для полной системы таких операторов это приводит к соотношению (13) с $Q_{i k}=\delta_{i k}$ (коэффициентами при $F_{k}$ в контрчленах $F_{i}$ ).

Контрчленная операция $L$ в (П.1) выражается соотношением $L=K R^{\prime}$ через первичную "операцию вычитания" $K$ и “неполную $R$-операцию"

$$
R^{\prime}=1-\sum L_{\gamma}+\sum L_{\gamma} L_{\gamma^{\prime}}-\cdots,
$$


представляющую собой вычитания на все расходящиеся подграфы $\gamma$, затем на пары непересекаюшихся подграфов $\gamma, \gamma^{\prime}$ и т.д. В схеме MS операция $L$ отбирает только полюсы по $\varepsilon$, поэтому все константы ренормировки $Z$ и матрица $Q$ имеют вид $1+$ полюсы по $\varepsilon$.

Допустим, что речь идет о ренормировке системы типа (16), в которой некоторые операторы являются младшими, а другие - старшими. Схему $\mathrm{MS}^{\prime}$, о которой говорилось в разделах 3 и 4 , можно определить по аналогии с введенной в $[6] \widetilde{R}$-операцией. Она отличается от обычной схемы MS наличием вкладов младших операторов в контрчлены старших: по определению для любого младшего $F_{i}$ контрчлены (П.1) строятся по обычным правилам схемы MS, так же и для вкладов старших $F_{k}$ в контрчлены старших $F_{i}$. Однако в коэффициентах при младших $F_{k}$ в контрчленах старших $F_{i}$ операция $K^{\prime}$ схемы MS' отбирает полюсы и все вклады нулевого порядка по $\varepsilon$ (константы). Возможность введения такой схемы вычитаний с сохранением всех общих положений теории ренормировки, в частности локальности контрчленов, объясняется тем, что во вкладах "старшие $\rightarrow$ младшие" имеется "дополнительная малость" порядка $\varepsilon$ из-за формального исчезновения всех старших структур (8) при $\varepsilon \rightarrow 0$. Это приводит к тому, что не только полюсные по $\varepsilon$ вклады, как обычно, но и вклады порядка $\varepsilon^{0}=1$ при младших операторах имеют структуру локальных контрчленов [6].

Такое определение схемы $\mathrm{MS}^{\prime}$ обеспечивает выполнение соотношений (23). В соответствуюшей матрице смешивания $Q^{\prime}$, кроме полюсов по $\varepsilon$, появляются и вклады типа констант, но только в тех элементах $Q_{i k}$, у которых индекс $i$ соответствует старшему оператору, а индекс $k$ - младшему.

\section{Список литературы}

[1] П. А. Байков, В. А. Ильин. ТМФ. 1991. Т. 88. С. 163.

[2] S. A. Larin. Phys. Lett. 1993. V. B303. P. 113.

[3] Д. Коллинз. Перенормировка. М.: Мир, 1988.

[4] S. Adler, W. Bardeen. Phys. Rev. 1969. V. 182. P. 1517.

[5] A. Bondi, G. Curci, G. Paffuti, P. Rossi. Ann. Phys. 1990. V. 199. P. 268.

[6] А.Н. Васильев, М.И. Вязовский, С.Э. Деркачев, Н. А. Кивель. ТМФ. 1996. Т. 107. C. 27.

[7] Н. Н. Боголюбов, Д. В. Ширков. Введение в теорию квантованных полей. М.: Наука, 1984.

[8] A. Kennedy. J. Math. Phys. 1981. V. 22. Р. 1330; Л. В. Авдеев. ТМФ. 1984. Т. 58. С. 308; А. Н. Васильев, С. Э. Деркачев, Н. А. Кивель. ТМФ. 1995. Т. 103. С. 179.

[9] А.Н. Васильев. ТМФ. 1989. Т. 81. С. 336.

[10] S. G. Gorishny, A. L. Kataev, S. A. Larin, L. R. Surguladze. Phys. Lett. 1991. V. B256. P. 81.

Поступила в редакцию 14.III.1996 г.

\section{I. Vyazovski, N. A. Kivel \\ ANALYSIS OF CHIRAL ANOMALY IN DIMENSIONAL REGULARIZATION BY MEANS OF PROJECTION TECHNIQUE}

We suggest a selfconsistent method for calculation of the chiral anomaly in the dimensional regularization. The advantage of our approach is the absence of the four dimensional symbols $\gamma_{5}$ and $\varepsilon_{\mu \nu \lambda \rho}$ in noninteger space-time dimensions. As an example we discuss the massless quantum electrodynamics. 